\title{
Planar Preprocessing for Spring Embedders
}

\author{
J. Joseph Fowler and Stephen G. Kobourov ${ }^{\star}$ \\ Department of Computer Science \\ University of Arizona
}

\begin{abstract}
Spring embedders are conceptually simple and produce straight-line drawings with an undeniable aesthetic appeal, which explains their prevalence when it comes to automated graph drawing. However, when drawing planar graphs, spring embedders often produce non-plane drawings, as edge crossings do not factor into the objective function being minimized. On the other hand, there are fairly straight-forward algorithms for creating plane straight-line drawings for planar graphs, but the resulting layouts generally are not aesthetically pleasing, as vertices are often grouped in small regions and edges lengths can vary dramatically. It is known that the initial layout influences the output of a spring embedder, and yet a random layout is nearly always the default. We study the effect of using various plane initial drawings as an inputs to a spring embedder, measuring the percent improvement in reducing crossings and in increasing node separation, edge length uniformity, and angular resolution.
\end{abstract}

\section{Introduction}

Some of the most flexible algorithms for drawing simple undirected graphs belong to a class known as force-directed algorithms. Also called spring embedders, such algorithms compute the layout of a graph using only information contained within the structure of the graph itself, rather than relying on domain-specific knowledge. Graphs drawn with such algorithms tend to be symmetric and have uniform edge lengths with good vertex distribution. As these are often desirable goals for the readability of a graph [18], it is no surprise that force-directed algorithms are the most commonly available tool for drawing graphs, networks, and diagrams. From family trees drawn by amateur genealogists to chemical molecules and Facebook friendship networks, the overwhelming majority of graphs that are drawn with such tools are small and sparse.

In general, force-directed methods define an objective function which maps each graph layout into a number in $\mathbb{R}^{+}$representing the energy of the layout. This function is defined so that low energies correspond to layouts in which adjacent nodes are near some pre-specified distance from each other, and in which non-adjacent nodes are wellspaced. Typically, force-directed algorithms are initialized with a random layout and iteratively move vertices so as to find a (often local) minimum of the objective function. Therefore, different initial layouts can influence the quality of the final layouts.

If the input graph happens to be planar, force-directed methods may reduce the edge crossings present in the initial random layout, although the objective function does not explicitly consider them. At the same time it is easy to compute an initial plane drawing in linear time and on an integer grid [1619]. Even for planar graphs, the running time

\footnotetext{
* Research supported by NSF grant CCF-1115971 and a grant from the Humboldt Foundation.
} 
of most force-directed algorithms is quadratic given that all vertex pairs are considered. Hence, adding an efficient preprocessing step that computes a plane (instead of random) initial layout, would not significantly increase the overall running time of a standard force-directed tool. We study the effect of such planar preprocessing on the number of crossings, node separation, edge lengths, and angular resolution.

\subsection{Related Work}

An early force-directed method by Eades [8] models edges as springs obeying Hooke's Law. A popular variant is that of Fruchterman and Reingold (FR) [11], who model the problem in terms of a strong nuclear force attracting two protons within the atomic nucleus at close range, but with an electrical force repelling them at a further range. Alternatively, forces between the nodes can be computed based on their graph theoretic distances, determined by the lengths of shortest paths between them. The algorithm of Kamada and Kawai (KK) [15] uses spring forces proportional to graph theoretic distances, and is in effect a variant of multi-dimensional scaling (MDS).

Modifications to the basic force-directed functionality, with the aim of improving the layout quality for planar graphs, have also been considered. Harel and Sardas [14] use an initial plane embedding and then apply simulated annealing while not introducing any crossings. They use an $O\left(n^{2}\right)$-time heuristic to find a large face as the outerface and an $O\left(n^{3}\right)$-time step to "center" the vertices in the drawing. Overall this method significantly improves the aesthetic quality of the planar layout, but at the expense of a significant increase in running time.

Bertault [1] describes a force-directed algorithm, PrED, that avoids introducing edge crossings but increases the running time to $O\left(n^{2}+n m\right)$ per iteration, while also requiring many more iterations to reach a low Tunkelang [20] studies a variant of the Fruchterman-Reingold method using quad trees to approximate forces at a distance in order to compute a gradient, which took $O(m+n \log n)$ time.

Brandes and Pich [4] show that layouts obtained with MDS are better at preserving relative distances than those obtained by force-directed algorithms. While they do not consider the number of edge crossings explicitly, one could argue that an algorithm that better preserves distances in the graph could lead to fewer edge crossings overall.

Picking least betweenness edges (in the fewest number of shortest paths), van Ham and Wattenberg [13] extracted a planar subgraph from their real-world small-diameter graph. A straight-line force-directed algorithm yields a layout with clearly separated clusters with high betweenness edges drawn with curved arcs.

Didimo et al. [6] customized a force-directed algorithm in order to reduce crossings of polyline drawings. They restricted forces acting on vertices to prevent new crossings, while allowing the topology to be altered when original crossings are eliminated. Dwyer et al. [7] used $P$-stress, a bend-point invariant goal function, to construct a topology preserving constrained graph layout where edges bend like rubber bands.

\section{Hypothesis and Methodology}

There are many specialized drawing algorithms that guarantee crossings-free layouts for certain classes of graphs: binary trees, arbitrary trees, outerplanar graphs, general 
planar graphs. However, most popular graph visualization tools (e.g., GraphViz, yEd, Gephi) do not contain implementations of such algorithms but rather use a spring embedder and do not test whether the input graph is planar. At the same time, many realworld examples (e.g., molecules in chemistry, small business hierarchies, genealogical relationships) are indeed planar and spring embedders are known for failing to find crossings-free layouts.

Our main goal is to gauge to what degree, for a given planar graph, can a plane embedding preprocessing step reduce the number of edge crossings in the final drawing. Similarly, we would like to assess the effect, if any, the preprocessing step has on node separation, edge lengths and angular resolution. It is generally believed that the quality of the final layout of a spring embedder can be improved by starting with a "better" layout than a random one, but there are no results on the effect that such preprocessing has on reducing the number of crossings while maintaining other aesthetic qualities. With this in mind, we took three standard planar layout algorithms and four off-theshelf spring embedders and tried to study these effects.

Data-sets: We use six libraries of connected planar graphs for our experiments:

I Prüfer trees (PRUFER) a set of uniformly sampled labeled trees obtained by using Prüfer's algorithm [17];

II Random trees (R-TREES) a set of random trees with unbounded maximum degree constructed by adding new pendant edges to randomly selected nodes in the tree constructed so far;

III Fusy graphs (FUSY) a set of uniformly sampled unlabeled graphs created using the Fusy generator [12];

IV Expansion graphs (EXPAN) a set of random triconnected planar graphs using the expansion method that performs $n-4$ split operations (starting with a $K_{4}$ ) on randomly selected nodes that randomly distributes neighbors between split nodes;

V Rome graphs (ROME) a subset of the undirected Rome graphs from the GDToolkit consisting of all connected planar graphs; and

VI AT\&T graphs (AT\&T) a subset of the directed AT\&T graphs (also known as the Graph Catalog) consisting of all connected planar graphs.

Libraries IIV were constructed to each have 10 graphs per value $n$, whereas, $\mathrm{V}$ and VI have a variable number of graphs per value of $n$; see Table 1 . Both tree libraries $\square$ and

Table 1. Data-set statistics for the $n$-vertex, $m$-edge graphs in each library

\begin{tabular}{|c|c|c|c|c|c|c|c|c|c|c|c|c|c|c|c|c|c|c|}
\hline \multirow{2}{*}{ Library } & \multirow{2}{*}{ Size } & \multicolumn{9}{|c|}{$n$} & \multicolumn{1}{|c|}{$m$} & \multicolumn{6}{c|}{ Average Degree Percentile } \\
\cline { 3 - 6 } & & $\min$ & $\max$ & avg & med & std & $\min$ & $\max$ & avg & med & std & $0 \%$ & $5 \%$ & $25 \%$ & $50 \%$ & $75 \%$ & $95 \%$ & $100 \%$ \\
\hline PRUFER & 910 & 10 & 100 & 55.0 & 55 & 26.3 & 9 & 99 & 54.0 & 54 & 26.3 & 1.80 & 1.86 & 1.94 & 1.96 & 1.97 & 1.98 & 1.98 \\
R-TREES & 910 & 10 & 100 & 55.0 & 55 & 26.3 & 9 & 99 & 54.0 & 54 & 26.3 & 1.80 & 1.86 & 1.94 & 1.96 & 1.97 & 1.98 & 1.98 \\
FUSY & 910 & 10 & 100 & 55.0 & 55 & 26.3 & 11 & 230 & 116.7 & 116 & 58.7 & 2.20 & 3.60 & 4.00 & 4.21 & 4.35 & 4.53 & 4.79 \\
EXPAN & 910 & 10 & 100 & 55.0 & 55 & 26.3 & 18 & 240 & 126.0 & 126 & 61.9 & 3.60 & 4.19 & 4.43 & 4.55 & 4.67 & 4.81 & 5.18 \\
ROME & 3279 & 10 & 89 & 25.8 & 24 & 11.8 & 9 & 103 & 30.2 & 28 & 14.2 & 1.80 & 2.00 & 2.17 & 2.32 & 2.46 & 2.77 & 3.85 \\
AT\&T & 854 & 10 & 100 & 29.0 & 23 & 19.4 & 9 & 120 & 32.7 & 26 & 21.3 & 1.80 & 1.85 & 2.00 & 2.22 & 2.47 & 3.00 & 4.74 \\
\hline
\end{tabular}


have the lowest median average degree of 1.96, while the other two constructed libraries III and IV have higher median average degrees of 4.21 and 4.55, respectively, whereas the pre-existing libraries $[\mathrm{V}]$ and $\mathrm{VI}$ also have low median average degrees of 2.32 and 2.22, respectively. Hence, libraries $\Pi, \Pi, \mathrm{V}$, and VI all have relatively low edge-density, while III and IV both have relatively high edge-density.

Tools: While there are many popular graph drawing tools (e.g., GraphViz, yEd, Gephi), most of them do not contain algorithms for planar graphs. With this in mind, we use the Open Graph Drawing Framework (OGDF) [5], together with additional code and scripts to generate libraries $\amalg$ IV to compute the graph aesthetics metrics, and to perform the experiments. Our code is written in $\mathrm{C}++$ and is available, along with all the graph libraries and experimental data, at http://planarpreprocessing.cs.arizona.edu. All figures with plots were drawn using gnuplot using the 'acspline' smoothing function.

Initial Placers: In addition to our hand-coded purely random layout (RANDOM), OGDF includes the following three straight-line drawing algorithms for planar graphs:

(i) FPPLayout (FPP) implements the algorithm of de Fraysseix, Pach and Pollack [10] for plane drawing on a grid of size $(2 n-4) \times(n-2)$, obtained by augmenting the graph until it is fully triangulated and then computing a canonical ordering and incrementally placing the vertices in this order;

(ii) SchnyderLayout (SCHNYDER) implements the algorithm of Schnyder [19] for plane drawing on an integer grid of size $(n-2) \times(n-2)$, obtained by augmenting the graph until it is fully triangulated, partitioning the interior edges into three trees and computing barycentric coordinates for the vertices;

(iii) PlanarStraightLayout (KANT) implements an improved version of the algorithm of Kant [16] for plane drawing on a grid of size at most $(2 n-4) \times(n-2)$, obtained by augmenting the graph until it is triconnected and then using an appropriate modification of the FPP algorithm to compute coordinates for the vertices.

Using RANDOM as an initial placer is equivalent to have no preprocessing done (given that is what most spring embedders use by default), while using any of the other placers constitutes adding a preprocessing step.

Embedders: OGDF also includes the following four distinct energy-based spring embedding algorithms for general graphs:

(1) SpringEmbedderFR (FR) implements the force-directed layout algorithm by Fruchterman-Reingold [11] using a grid-variant of the algorithm to speed up the computation of repulsive forces (using the defaults: (i) 400 iterations, (ii) has small initial random perturbations, (iii) minimum distance of 20 between connected components, and (iv) automatic scaling),

(2) SpringEmbedderFRExact (FRE) provides an alternative implementation of the FR spring embedder with exact force computations (using the defaults: (i) 1000 iterations, (ii) has small initial random perturbations, (iii) minimum distance of 20 between connected components, (iv) cooling factor of .9, (v) ideal edge length of 10 , and (v) tolerance factor of 0.01 , which the fraction of ideal length below which convergence is achieved); 
(3) SpringEmbedderKK (KK) implements that MDS spring embedder of Kamada and Kawai [15] (using the defaults: (i) stop tolerance of 0.0001 for energy level, (ii) maximum number of iterations is based on input graph, (iii) all edges have equal length, and (iv) desirable edge length is proportional to the area of the display grid divided by the maximum distance between two nodes in initial layout); and

(4) StressMajorization (SM) implements simple stress majorization by Pich [3] that allows radial constraints based on shortest path distances (using the defaults: (i) 50 iterations and (ii) automatic scaling).

Aesthetics: In addition to counting crossings $\mathfrak{c}$ of $G$, we consider the following three aesthetic parameters for a drawing of a graph on $n$ nodes and $m$ edges, which have been shown to have an effect upon human understanding of a graph; see e.g., Purchase [18]:

(a) Minimum angle aesthetic metric $\mathfrak{m a}$ from [18], is based on $d_{m a}$, the average deviation of adjacent incident edge angles from the ideal minimum angle:

$$
d_{m a}=\frac{1}{n} \sum_{i=1}^{n}\left|\frac{\vartheta_{i}-\theta_{i \min }}{\vartheta_{i}}\right|
$$

where $\vartheta_{i}$ is the ideal minimal angle at the $i$ th node, namely $\vartheta_{i}=\frac{360^{\circ}}{\operatorname{degree}\left(v_{i}\right)}$ and $\theta_{i \text { min }}$ is the actual minimum angle between the incident edges at the $i$ th node. Then $\mathfrak{m a}$ is defined as $\mathfrak{m a}=1-d_{m a}$ so that $0 \leq \mathfrak{m a} \leq 1$, where $\mathfrak{m a}=0$ indicates that every node in the drawing has a zero minimum angle (i.e., two overlapping incident edges) and $\mathfrak{m a}=1$ indicates "perfect angular resolution" in which case every angle is optimal as in Lombardi drawings.

(b) Edge lengths aesthetic metric $\mathfrak{e l}$ is based on $d_{e l}$, the average percent deviation of edge lengths using a mean central tendency:

$$
d_{e l}=\frac{1}{m} \sum_{j=1}^{m}\left|\frac{\left|e_{j}\right|-|e|_{\text {avg }}}{\max \left\{|e|_{\text {avg }},|e|_{\max }-|e|_{\text {avg }}\right\}}\right|
$$

where $\left|e_{j}\right|$ is the length of the $j$ th edge, $|e|_{\text {avg }}$ is the average edge length, and $|e|_{\max }$ is the maximum edge length. Dividing by the $\max \left\{|e|_{\text {avg }},|e|_{\max }-|e|_{\text {avg }}\right\}$ guarantees that $0 \leq d_{e l} \leq 1$, so that $\mathfrak{e l}=1-d_{e l}$ will be bounded $0 \leq \mathfrak{e l} \leq 1$, where $\mathfrak{e l}=0$ could indicate that half the edges were all short while other half of the edges were all long while $\mathfrak{e l}=1$ indicates all the edges have the same length.

(c) Node separation aesthetic metric $\mathfrak{n s}$ is based on $d_{s r}$, the average percent deviation of separating rectangles 1 using a mean central tendency:

$$
d_{s r}=\frac{1}{n} \sum_{i=1}^{n}\left|\frac{\left|v_{i}\right|-|v|_{\text {avg }}}{\max \left\{|v|_{\text {avg }},|v|_{\text {max }}-|v|_{\text {avg }}\right\}}\right|
$$

\footnotetext{
${ }^{1}$ These are the $n$ axis-aligned, smallest-perimeter rectangles contained within the bounding box of all nodes, such that each side of the rectangle is either incident to the neighbor(s) of its node or coincides with a side of the bounding box.
} 
where $\left|v_{i}\right|$ is the perimeter of the smallest rectangle separating the ith node from its nearest neighbors; $|v|_{\text {avg }}$ is the average over all nodes; and $|v|_{\max }$ is the maximum over all nodes. Given that $0 \leq d_{s r} \leq 1$, then $\mathfrak{n s}=1-d_{s r}$ will also be bounded $0 \leq \mathfrak{n} \mathfrak{s} \leq 1$. Then $\mathfrak{n s}=0$, could indicate that half the nodes were relatively close to their nearest neighbors while the other half of the nodes were far from their nearest neighbors, whereas $\mathfrak{n} \mathfrak{s}=1$ indicates the nodes are more uniformly distributed.

General Methodology. For each graph used in our experiments, we evaluated the above four aesthetic metrics on layouts produced by each of the four initial placers and by each placer/embedder pairs, where the placer-generated layout is used as the initial layout for the embedder; see Table 2 To reduce the noise of our data, we ran each placer not once,

Table 2. Sample layouts of a representative 55-node graph with 121 edges from EXPAN library where each row uses a different placer and each column uses a different spring embedder (except for the first column using only the placer)

Initial Placer


but five times, to generate five distinct layouts of the same graph, which we then treated as a separate data point (i.e., as though each of the five layouts were from five different graphs). To obtain a value for a particular aesthetic metric, we took the median of the metric over all layouts. When comparing the results of using a planar preprocessor, we took the median of the percent reduction in crossings between each pair of layouts before and after the preprocessing step was included.

\section{Experiments and Results}

For each of the graph libraries, and for each spring embedder (FR, FRE, KK, SM) we ran experiments to determine the effect of planar preprocessing (using FPP, SCHNYDER, or KANT), compared against using RANDOM as an initial placer, on the aesthetic criteria (crossings, edge length, node separation, angular resolution). We first analyze the placers and embedders separately, before turning to combining the two.

Placer Aesthetics. Figure 1 provides a comparison between the four initial placers. Lower $y$-values for the left column of plots indicates fewer crossings, which is desirable. Higher $y$-values for the remaining three columns indicate more desirable aesthetic values for minimum angles, edge lengths, and node separation. As the only non-planar embedder, RANDOM has the highest number of crossings. It also has the worst angular resolution, but provides the best edge length and node separation. KANT has the best angular resolution and is consistently second in the other metrics, with $15-30 \%$ better angular resolution and about $20 \%$ more uniform edge lengths and node separation than the other planar placers. SCHNYDER and FPP have nearly identical aesthetics (within 5\%) for all the graphs, with the first slightly better with angular resolution and node separation, and the second better with edge lengths. In summary, among the three planar placers, KANT has the best overall aesthetics.

Embedder Aesthetics. Figure 2 provides a comparison between the spring embedders, when using RANDOM as an initial placer. With no preprocessing, $\mathrm{KK}$ has the least number of crossings producing almost planar layouts for the tree and AT\&T libraries. FR and FRE have similar performance, except that the exact variant produces $25-50 \%$ fewer crossings across all six libraries. SM performs noticeably worse than the other three with up to $40 \%$ worse angular resolution and up to $25 \%$ worse edge lengths and node separation (although it is the fastest among the embedders). In summary, among the four embedders, $\mathrm{KK}$ has the best overall aesthetics when no preprocessing is done.

Brandenburg et al. [2] experimentally compared straight-line graph drawing algorithms including variants of FRE and KK implemented in the GraphED framework. Their results match ours in that KK is in general superior to FRE in terms of edge lengths and node distribution. Their variant of KK was efficient, but only performs $10 * n$ iterations, rather than iterating until the energy function converges to a preset threshold as is the case in the standard $\mathrm{KK}$ algorithm used in OGDF. While $10 * n$ iterations may be suffice for small sparse graphs considered in [2], this is not the case in general. For instance, for denser graphs in the FUSY and EXPAN libraries with a 100 edges, we found FRE to be up to 20 times faster than KK. 
Effect of Planar Preprocessing with KANT. Figure 3 shows the percent improvement in each aesthetic metric when KANT is used for planar preprocessing. The $x$-axis shows the number of vertices and the $y$-axis shows either the median number of crossings or the median of the normalized 0-to-1 value for the other metrics. High $y$-values in the left column indicate more crossing reduction, which is desirable. High $y$-values or near-zero $y$-values in the remaining columns indicate consistent improvement or no discernible effect with regard to that column's metric.

Preprocessing with KANT clearly reduced crossings. Moreover, this improvement does not come at the cost of negatively impacting any of the other aesthetic metrics.

Aesthetic Comparison for the Initial Placers
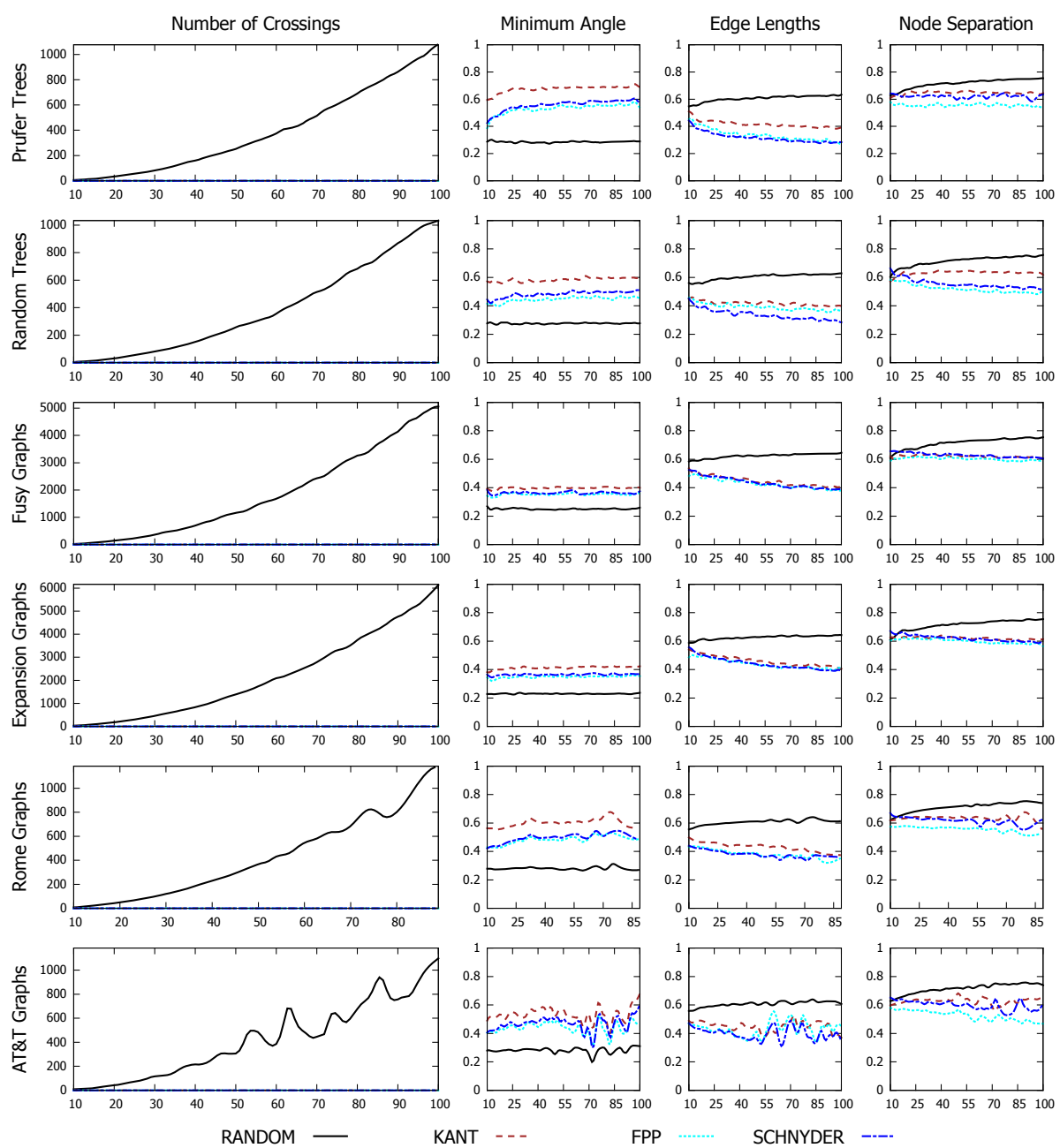

Fig. 1. Median of aesthetic metrics vs. number of nodes for each initial placer where values closer to 1 are better for the right three columns 
From the last three columns in Fig. 3, we can see a remarkable lack of degradation in any of the three metrics for FR and FRE. The only exception is SM which shows worse angular resolution (which was already the worst even without preprocessing) 2 The effect of preprocessing is negligible for KK. In summary, using KANT as a preprocessing step for FR and FRE resulted in significant reduction in crossings, while not negatively impacting the other aesthetics.

Aesthetic Comparison of the Spring Embedders using RANDOM as Initial Placer
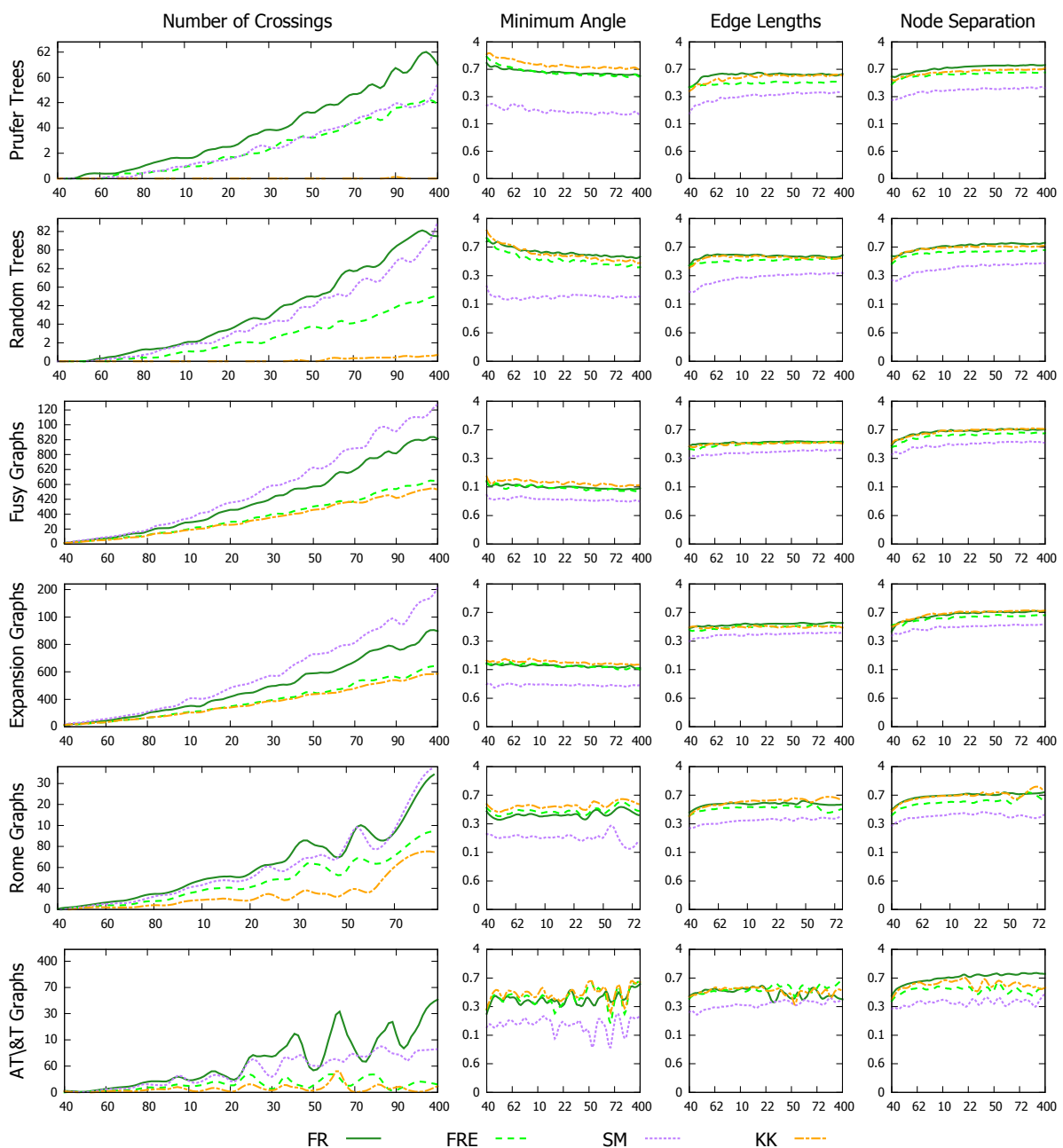

Fig. 2. Median of aesthetic metrics vs. number of nodes for each spring embedder using random layout where values closer to 1 are better for the right three columns

\footnotetext{
${ }^{2}$ It is worth noting here that SM itself is more of a pre-processing step than a standard embedder.
} 
Specifically, our results indicate that KANT+FRE is the best placer+embedder pair. In fact, the combination is good enough to compare directly with the best (but much more expensive) $\mathrm{KK}$ embedder. The two approaches yield very similar results for all our graph libraries. KK is slightly better with 5 to 10 percent fewer crossings. On the other hand, KANT+FR is slightly better with 5 to 10 percent better edge lengths and node separation. Both approaches provide nearly identical minimum angle metrics; the full version of this paper [9] provides a figure directly comparing KANT+FRE to KK.

We would like to point out that most spring embedders currently in use are based on implementations very similar to FR and FRE, as implementations of KK are signif-

Aesthetic Percent Improvement using KANT as Initial Placer
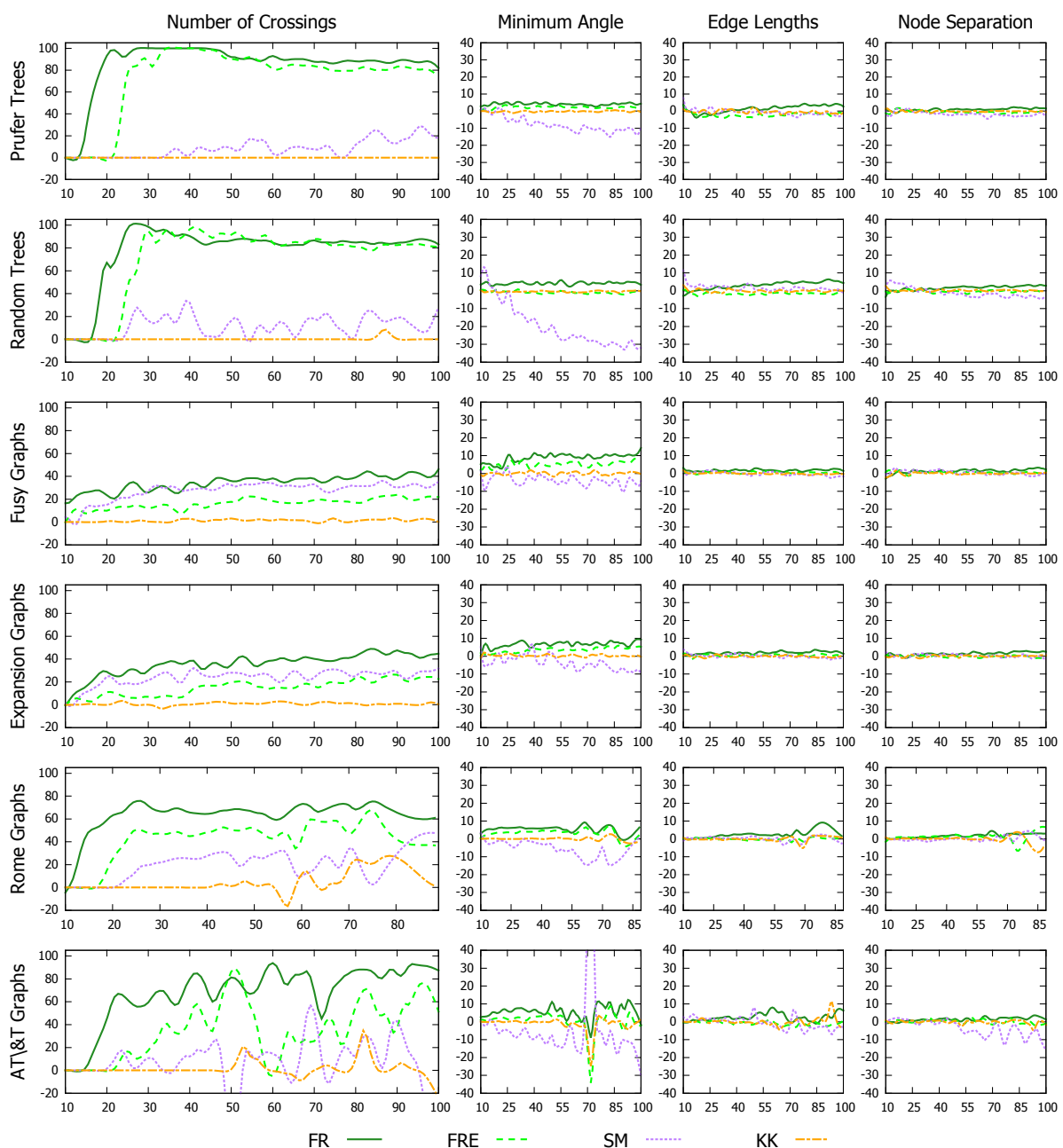

Fig. 3. Percent improvement in aesthetic metrics vs. number of nodes for spring embedders using KANT where higher, non-negative percentage is generally better 
icantly more computationally expensive. Our experiments indicate that popular spring embedders might obtain nearly as good results as KK for planar graphs, with the help of a linear-time planar preprocessing step such as KANT.

Effect of Planar Preprocessing with FPP and SCHNYDER. We detail our results for FPP and SCHNYDER here, while also providing figures in [9] analogous to Figure 3] for both FPP and SCHNYDER that show the percent improvement in each aesthetic metric when FPP and SCHNYDER are used for planar preprocessing.

For FPP, both FR and FRE had crossings reduced significantly for all graphs with 20 vertices or more. In particular, crossings were reduced by at least $80 \%$ for the PRUFER and R-TREES libraries, 40\% for the ROME and AT\&T libraries, and 30\% for the FUSY and EXPAN libraries, which we see as a non-trivial improvement.

Preprocessing with SCHNYDER reduces crossings as well as FPP (given their similarity in Fig. 10 maintaining the $80 \%$ reduction for the trees libraries and the 30 to $40 \%$ reduction for the other four libraries. Even though SCHNYDER had a small advantage in angular resolution over using FPP when preprocessing the FUSY and EXPAN libraries, KANT also showed a similar 5-10\% improvement in increasing minimum angles for those two libraries; see Fig. 3. In summary, while FPP, SCHNYDER, and KANT have similar non-crossing aesthetic metrics, KANT's overall stronger performance in uniformly reducing crossings clearly demonstrates that KANT is a better choice than either FPP or SCHNYDER for planar preprocessing.

\section{Conclusion and Future Work}

We described a set of experiments on the effect of using three standard planar embedding algorithms as a preprocessing step for four off-the-shelf force-directed algorithms. We use default parameters and treat all algorithms as black-boxes; i.e. no attempt was made to adjust parameters, knowing that the other algorithm will be used (or vice versa).

Surely, we could have guaranteed crossings-free drawings by using specialized algorithms designed for trees and planar graphs. But precisely because these algorithms are specialized, they are very rarely used! Instead, the vast majority of graph drawings are obtained with the default setting of popular spring embedders such as GraphViz, yEd, Gephi, etc. Adding a planar embedding preprocessing step to such existing tools has little impact on the running time for any input graph, but offers an increased likelihood of obtaining a plane layout, or reducing the crossings, if the input graph is planar.

Specifically, our results indicate that preprocessing small planar graphs reduces the number of crossings most notably for the Fruchterman-Reingold force-directed layout algorithms, while not significantly impacting any of the aesthetic metrics we measured. There was little or no effect on any of the metrics when planar preprocessing was combined with a Kamada-Kawai force-directed algorithm. While not particularly surprising, to the best of our knowledge, these effects had not been experimentally verified before. We consider this a promising preliminary study.

The results lead to a few follow-up questions. First, it is very likely that integrating the preprocessing and force-directed steps will lead to better results. Specifically, is it possible to coordinate the ideal-edge-length of the spring embedder with the average edge length produced by the planar embedding? Can adjusting the cooling schedule (to 
ensure no big moves are made) make significant impact on the final drawing? Second, what is the impact of planar preprocessing for larger graphs? While KANT+FRE and KK had comparable aesthetics for graphs with under 100 vertices, KK becomes impractical for larger graphs, requiring minutes to produce a layout. If the performance for large graphs remains as good, KANT+FRE might be the better option.

\section{References}

1. Bertault, F.: A force-directed algorithm that preserves edge-crossing properties. Information Processing Letters 74(1-2), 7-13 (2000)

2. Brandenburg, F.J., Himsolt, M., Rohrer, C.: An Experimental Comparison of Force-Directed and Randomized Graph Drawing Algorithms. In: Brandenburg, F.J. (ed.) GD 1995. LNCS, vol. 1027, pp. 76-87. Springer, Heidelberg (1996)

3. Brandes, U., Pich, C.: More Flexible Radial Layout. In: Eppstein, D., Gansner, E.R. (eds.) GD 2009. LNCS, vol. 5849, pp. 107-118. Springer, Heidelberg (2010), http://dx.doi.org/10.1007/978-3-642-11805-0_12

4. Brandes, U., Pich, C.: An Experimental Study on Distance-Based Graph Drawing (Extended Abstract). In: Tollis, I.G., Patrignani, M. (eds.) GD 2008. LNCS, vol. 5417, pp. 218-229. Springer, Heidelberg (2009)

5. Chimani, M., Gutwenger, C., Jünger, M., Klau, G., Klein, K., Mutzel, P.: The open graph drawing framework. In: Handbook of Graph Drawing and Visualization

6. Didimo, W., Liotta, G., Romeo, S.A.: Topology-Driven Force-Directed Algorithms. In: Brandes, U., Cornelsen, S. (eds.) GD 2010. LNCS, vol. 6502, pp. 165-176. Springer, Heidelberg (2011)

7. Dwyer, T., Marriott, K., Wybrow, M.: Topology Preserving Constrained Graph Layout. In: Tollis, I.G., Patrignani, M. (eds.) GD 2008. LNCS, vol. 5417, pp. 230-241. Springer, Heidelberg (2009)

8. Eades, P.: A heuristic for graph drawing. Cong. Numerantium 42, 149-160 (1984)

9. Fowler, J., Kobourov, S.G.: Planar preprocessing for spring embedders. Technical Report TR12-03, Dept. of Computer Science, Univ. of Arizona (2012)

10. de Fraysseix, H., Pach, J., Pollack, R.: How to draw a planar graph on a grid. Combinatorica 10(1), 41-51 (1990)

11. Fruchterman, T., Reingold, E.: Graph drawing by force-directed placement. Software - Practice and Experience 21(11), 1129-1164 (1991)

12. Fusy, É.: Uniform random sampling of planar graphs in linear time. Random Structures and Algorithms 35(4), 464-522 (2009)

13. van Ham, F., Wattenberg, M.: Centrality based visualization of small world graphs. Comput. Graph. Forum 27(3), 975-982 (2008)

14. Harel, D., Sardas, M.: Randomized graph drawing with heavy-duty preprocessing. Journal of Visual Languages and Computing 6(3), 233-253 (1995)

15. Kamada, T., Kawai, S.: An algorithm for drawing general undirected graphs. Information Processing Letters 31, 7-15 (1989)

16. Kant, G.: Drawing planar graphs using the canonical ordering. Algorithmica 16, 4-32 (1996)

17. Prüfer, H.: Neuer Beweis eines Satzes über Permutationen. Archiv für Mathematik und Physik, pp. 142-144 (1918)

18. Purchase, H.: Metrics for graph drawing aesthetics. Journal of Visual Languages \& Computing 13(5), 501-516 (2002)

19. Schnyder, W.: Embedding planar graphs on the grid. In: Proceedings of the 1st ACM-SIAM Symposium on Discrete Algorithms (SODA), pp. 138-148 (1990)

20. Tunkelang, D.: JIGGLE: Java Interactive Graph Layout Environment. In: Whitesides, S.H. (ed.) GD 1998. LNCS, vol. 1547, pp. 413-422. Springer, Heidelberg (1999) 